This article has been accepted for publication in a future issue of this journal, but has not been fully edited.

Content may change prior to final publication in an issue of the journal. To cite the paper please use the doi provided on the Digital Library page.

\title{
Graphical Modelling of Pinched Hysteresis Loops of Memristors
}

\author{
Xiao-Meng Wang ${ }^{1}$, Shu-Yuen Ron Hui ${ }^{2 *}$ \\ 1 Department of Electrical \& Electronic Engineering, The University of Hong Kong, \\ Pokfulam, Hong Kong \\ ${ }^{2}$ Department of Electrical \& Electronic Engineering, The University of Hong Kong and \\ Imperial College London, London, U.K. \\ ronhui@eee.hku.hk
}

\begin{abstract}
In this paper, a graphical modelling approach of the pinched hysteresis loops exhibited by memristors is presented. This method provides a tool to emulate the hysteresis loop pinched at the origin, with the lobe area varying with the excitation frequency. The direction of the pinched hysteresis loop can be controlled. This graphical modelling method provides an alternative to describe the behaviour of memristors without deriving the coupled nonlinear differential equations typically required for physical memristors. The method has been successfully applied to model the Hewlett-Packard memristor device.
\end{abstract}

\section{Introduction}

Memristors postulated by Leon Chua in 1971 [1] was confirmed by a Hewlett Packard research team in 2008 [2]. Since then, research in memristors has regained momentum [3]-[9]. The first man-made memristor has recently been traced back [10] to the 1801 carbon arc experiment by Humphrey Davy [11]. The hysteresis loops exhibited by memristors are different from those occur in magnetic materials in at least two ways. Firstly, the memristor's hysteresis loop is always pinched at the origin. Secondly, the lobe area of the pinched hysteresis loop varies with excitation frequency.

There are several well-established modelling methods for the magnetic hysteresis loops. Among them are Jile-Atherton [12] and the Preisach model [13]. The advantage of the Preisach model is that it can easily be implemented in a graphical approach [14], [15], as demonstrated in many applications such as high frequency ferrite core analyses [16],[17]. The graphical approach has the advantage that the model does not require detailed physical equations and yet can still provide a computer-aided tool to predict the device behaviour. However, existing hysteresis modelling methods are not applicable to memristors. Although some memristors, such as discharge arc and lamps [18] can be described with coupled nonlinear differential equations or described in a state-space approach [19], there is no graphical method, (similar to the Preisach model for non-pinched hysteresis loops) available for general modelling of pinched hysteresis loops.

The major difference of a memristive device and non-memristive device is that the former exhibits a characteristic of "pinched hysteresis loop". The three fingerprints of a memristor are summarized [20] as:

1) Pinched hysteresis loop at origin 
This article has been accepted for publication in a future issue of this journal, but has not been fully edited.

Content may change prior to final publication in an issue of the journal. To cite the paper please use the doi provided on the Digital Library page.

2) Hysteresis lobe area decreases as frequency increases

3) Pinched hysteresis loop shrinks to a single-valued function at infinite frequency.

In this paper, a graphical method for suitable for modelling the three fingerprints of the pinched hysteresis loops of memristors is presented. The basic concept is explained and parameters that can alter the shapes and areas of the pinched loops are explained. The mathematic implementation of such method is illustrated. The application procedure and its implementations in physical examples are given in Section 5.

\section{Basic graphical approach to the modelling of pinched hysteresis loops}

In this section, the basic concept of the graphical method is explained. It will be shown later that several parameters have to be introduced in order to provide the 3 features of memristors.

\subsection{Periodic excitation function}

A periodic sinusoidal excitation function $\alpha$ is defined as:

$$
\alpha(j)=M \cdot \sin (\omega \cdot j \cdot \Delta T)
$$

where $M$ is the peak value of the periodic function, $j$ is the present sample position, $\omega$ is excitation angular speed and $\Delta T$ is selected sampling interval according to the simulation system defined sampling frequency. This sinusoidal function (Fig.1a) is used to illustrate the graphical method with four stages illustrated, although other periodic function can in principle be used. In this paper, this excitation function $\alpha(j)$ is the input to the memristor. the output response of the memristor is labelled as $\beta$. The pinched hysteresis loop can be formed by plotting $\beta$ against $\alpha$ over at least one excitation cycle. In the computation, the time variable of the excitation function is discretised. It increases in steps of a time increment of $\Delta T$. It takes $N$ time increments to complete one full cycle (i.e. $N=2 \pi /(\omega . \Delta T)$ ). Since the proposal method is a graphical approach, the response function is not determined by any analytical equation. Instead, it is obtained from the incremental area of a sliding diagram (Section 2.3). 
This article has been accepted for publication in a future issue of this journal, but has not been fully edited.

Content may change prior to final publication in an issue of the journal. To cite the paper please use the doi provided on the Digital Library page.
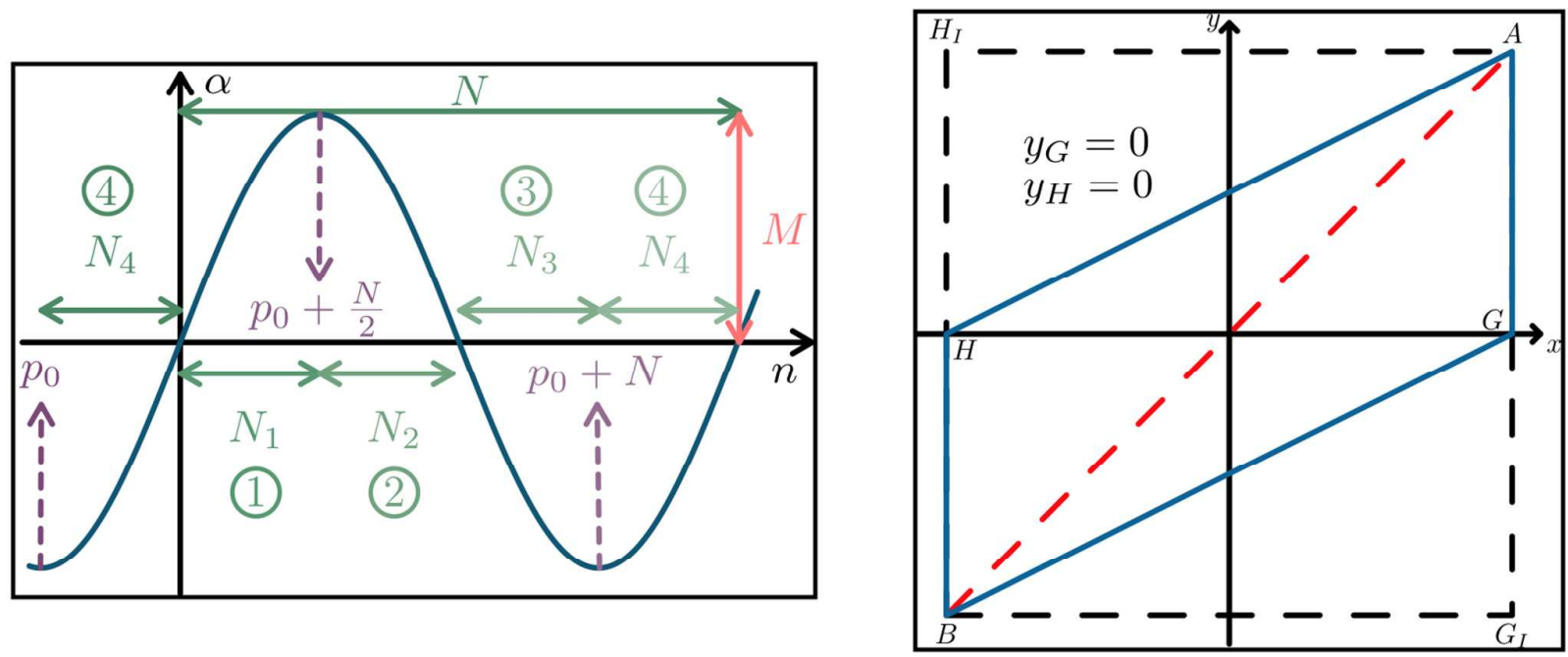

\section{$a$}

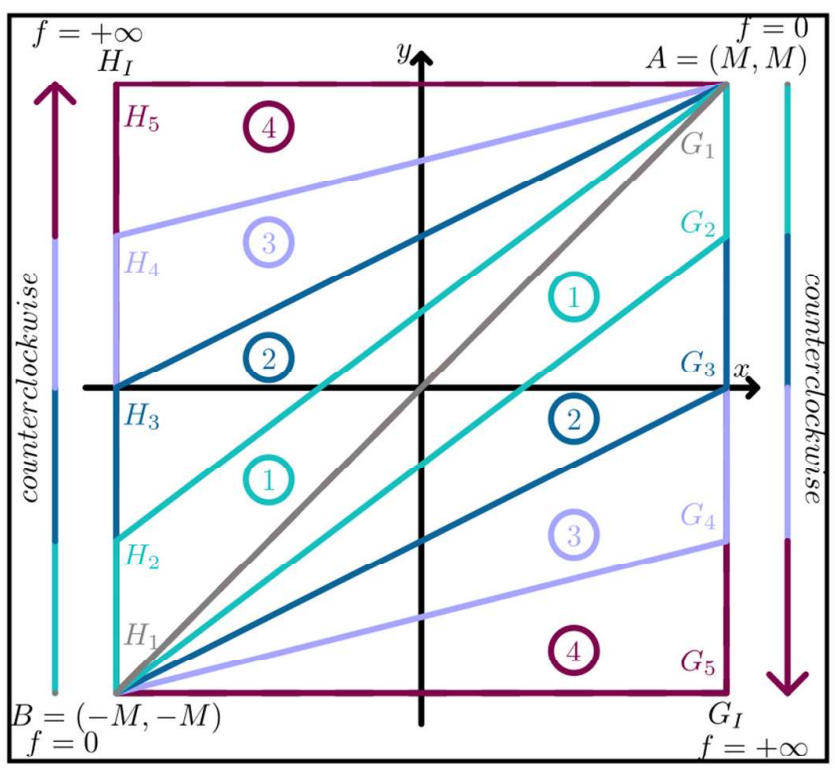

C

Fig. 1. Sinusoidal excitation and the mechanism of graphical approach

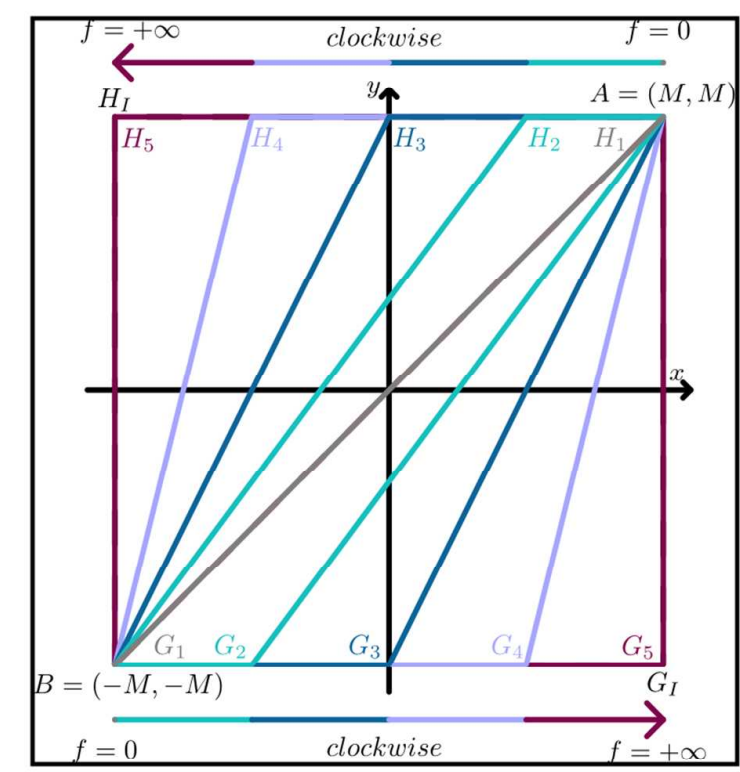

$d$

a Illustration of the sinusoidal periodic excitation

$\mathrm{b}$ A sliding diagram for the pinched hysteresis loop of memristor

c Progressive changes of the sliding diagram when $y_{G}$ changes from $M$ to $-M$ (for a "counter clockwise" loop in the first quadrant)

d Progressive changes of the sliding diagram when $x_{G}$ changes from $M$ to $-M$ (for a "clockwise" loop in the first quadrant)

\subsection{Graphical approach to the pinched hysteresis loop of memristor with loop direction control}

Apart from three fingerprints of a memristor, the loop direction is also an important characteristic of memristor. Thus, the graphical modelling method should cope with both clockwise (CW) and counter clockwise $(\mathrm{CCW})$ pinched hysteresis loops. To be consistent in this paper, the loop in the first quadrant of 
This article has been accepted for publication in a future issue of this journal, but has not been fully edited.

Content may change prior to final publication in an issue of the journal. To cite the paper please use the doi provided on the Digital Library page. the pinched hysteresis loop plot is used to specify the direction.

1) Sliding diagrams for pinched hysteresis loop with "counter clockwise" loop in the first quadrant:

A Cartesian coordinate system adopted in Fig.1b involves a dashed square $A H_{I} B G_{I}$ with its centre at the origin of the coordinate system. The length of each side of the square is $2 M$. Points $A$ and $B$ are two fixed points with the $\mathrm{x}-\mathrm{y}$ coordinates of $(M, M)$ and $(-M,-M)$ respectively. For a pinched hysteresis loop with "counter clockwise" direction in the first quadrant, the points $G$ and $H$ are two movable points that could slide "vertically" on the straight lines $A G_{I}$ and $B H_{I}$ respectively. Under such conditions, their positions are defined as $G=\left(x_{G}, y_{G}\right)$ where $x_{G}=M$, and $H=\left(x_{H}, y_{H}\right)$ where $x_{H}=-M$. This means they will slide "vertically". The dashed red line $A B$ is the diagonal line of $A H_{I} B G_{I}$ and it separates the square into two regions so that $G$ and $H$ would slide within their respective regions. The proposed graphical method requires the points $G$ and $H$ to slide along $A G_{I}$ and $B H_{I}$ respectively so that $A H$ and $B G$ are always in parallel.

The operation of the sliding diagram for $\mathrm{CCW}$ with the parallel relationship of $A H$ and $B G$ can be mathematically described by equations (2)-(4) as:

For a counter-clockwise (CCW) loop in the first quadrant of the pinched hysteresis loop,

$$
\begin{gathered}
\left\{\begin{array}{c}
-M \leq y_{G} \leq M \\
x_{G}=M
\end{array}\right. \\
\left\{\begin{array}{c}
-M \leq y_{H} \leq M \\
x_{H}=-M
\end{array}\right. \\
y_{G}=-y_{H}
\end{gathered}
$$

Equations (2) and (3) ensure that the points $G$ and $H$ slide along the vertical lines $A G_{I}$ and $B H_{I}$, respectively. Equation (4) makes sure that $B G$ and $A H$ are always in parallel. Fig. $1 \mathrm{~b}$ represents a sliding diagram complying with (2)-(5) when $y_{G}$ and $y_{H}$ are zero.

In order to illustrate the sliding function of the graphical method, Fig.1c is used to demonstrate the conditions of the sliding diagrams with $y_{G}$ changing from $M$ to $-M$. Fig. 1c shows the sliding diagram. The initial condition occurs when $y_{G}=M$ and $y_{H}=-M$ at $G_{1}$ and $H_{l}$. Under this condition, the sliding point $G$ overlaps with position $A$ and $H$ with position $B$. The straight lines $A H$ and $B G$ also overlap (as the "grey" straight line passing through the origin in Fig.1c). The area enclosed by the parallelogram $A G B H$ is zero.

As $y_{G}$ slides to from $\mathrm{M}$ to $0.5 \mathrm{M}$ and $y_{H}$ from $-\mathrm{M}$ to $-0.5 \mathrm{M}$ (according to (4)), points $\mathrm{G}$ and $\mathrm{H}$ move to the positions labelled as $G_{2}$ and $H_{2}$ in the sliding diagram. After these sliding movements, the two 
This article has been accepted for publication in a future issue of this journal, but has not been fully edited.

Content may change prior to final publication in an issue of the journal. To cite the paper please use the doi provided on the Digital Library page. straight lines $\mathrm{BG}_{2}$ and $\mathrm{AH}_{2}$ form two parallel lines as highlighted in the "green" lines labelled as "1". Now, a parallelogram $\mathrm{AG}_{2} \mathrm{BH}_{2}$ with positive area is formed.

As $y_{G}$ continues to slide from $0.5 \mathrm{M}$ to 0 and $y_{H}$ from $-0.5 \mathrm{M}$ to 0 , the points $\mathrm{G}$ and $\mathrm{H}$ move to new positions G3 and H3, respectively. Now a parallelogram increases its size to the area of $A G 3 B H 5$ as shown in the "blue" parallelogram labelled as " 2 ". When $y_{G}$ continues to slide from 0 to $-0.5 \mathrm{M}$ (and $y_{H}$ from 0 to $0.5 \mathrm{M})$, the area of the parallelogram increases to $A G_{4} B H_{4}$ as shown in the "purple" parallelogram labelled as " 3 ". The parallelogram $A G B H$ continues to expand until it forms a square $A G B H$ when $y_{G}=-M\left(y_{H}=M\right)$. The changes of $A G B H$ can be identified with their different colours.

\section{2) Sliding diagrams for pinched hysteresis loops with "clockwise" loop in the first quadrant:}

If the pinched hysteresis loop is "clockwise" in the first quadrant, the movable points $G$ and $H$ will slide "horizontally" along the horizontal lines $B G_{I}$ and $A H_{I}$, respectively. Under these conditions, $G=\left(x_{G}, y_{G}\right)$ where $y_{G}=-M$, and $H=\left(x_{H}, y_{H}\right)$ where $y_{H}=M$. This leads to the following equations for the "clockwise" loop:

For a clockwise loop in the first quadrant of a pinched hysteresis loop,

$$
\left\{\begin{array}{c}
-M \leq x_{G} \leq M \\
y_{G}=-M
\end{array}\right.
$$

(5)

$$
\begin{aligned}
& \left\{\begin{array}{c}
-M \leq x_{H} \leq M \\
y_{H}=M
\end{array}\right. \\
& x_{G}=-x_{H}
\end{aligned}
$$

Equations (5) and (6) define the restricted area which $G$ and $H$ could slide; (7) enforces the parallel relationship between $A G$ and $B H$.

Besides the sliding direction of points $\mathrm{G}$ and $\mathrm{H}$, the same principle previously described for the "counter clockwise" loop in the first quadrant remains valid. The progressive changes of the sliding diagram for a "clockwise" hysteresis loop is shown in Fig.1d.

\subsection{Graphical approach to the formation of the pinched hysteresis loop}

With the introduction of the sliding diagrams in the previous section, it is necessary to explain the procedure for forming the pinched hysteresis loop. It should be noted that each pinched loop has both $\mathrm{CW}$ and $\mathrm{CCW}$ loops. A typical pinched loop inherits self-crossing feature. If it has a CCW loop in the first 
This article has been accepted for publication in a future issue of this journal, but has not been fully edited.

Content may change prior to final publication in an issue of the journal. To cite the paper please use the doi provided on the Digital Library page.

quadrant, then it has a CW loop in the third quadrant. Similarly, a pinched loop with a CW loop in the first quadrant will have a CCW loop in the third quadrant. The basic graphical method is designed to exhibit the pinched hysteresis loop self-crossing feature.

The proposed graphical modelling requires a function with an input $\alpha$ (representing the excitation) and an output $\beta$ (representing the loop magnitude). The excitation function is assumed to be a bipolar and periodic one such as the sinusoidal function in Fig.1a.

In this section, the specific operation details of a CCW loop in the first quadrant is presented first, two scenarios which are excitation increasing and decreasing conditions are discussed. The operation of the $\mathrm{CW}$ case will be addressed later.

\section{1) Response Generation of a CCW Loop}

When the instantaneous excitation value is increasing comparing with the previous value in a counter clockwise loop (i.e. $\frac{d \alpha(j)}{d j} \geq 0$ ), Fig. 6 shows a graphical approach to determine the output function $\beta$.

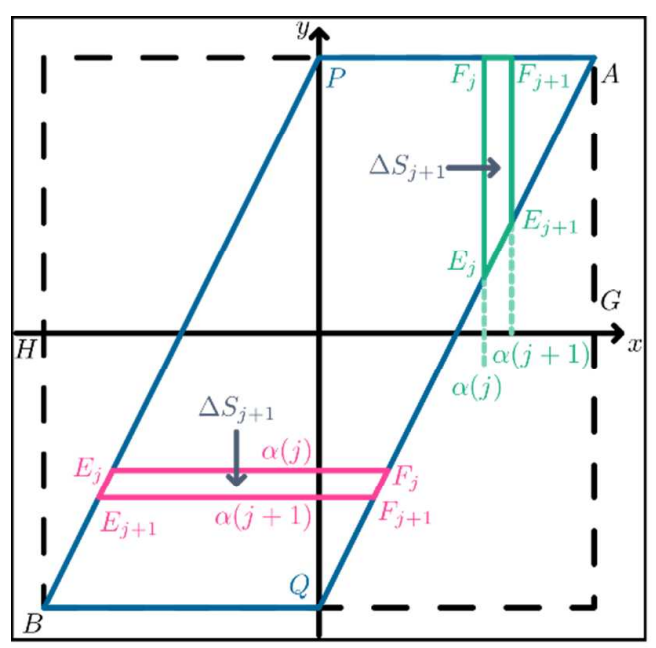

$a$

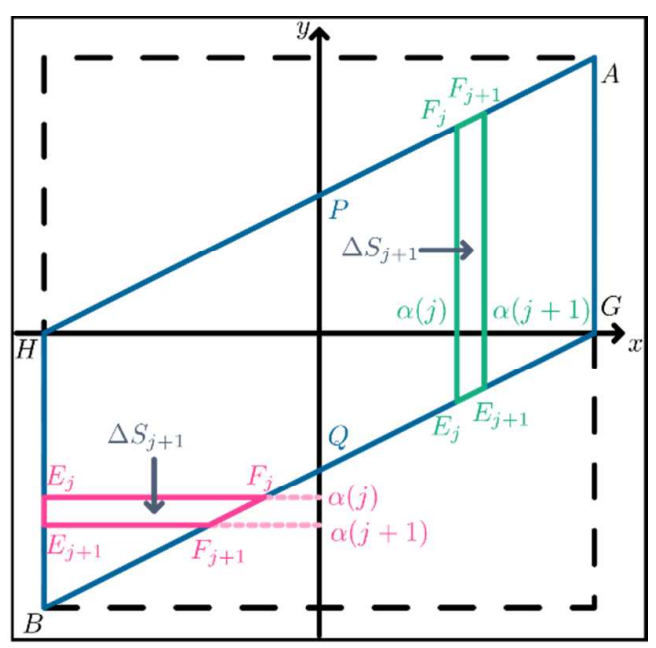

$b$

Fig. 2. Sliding diagram of the graphical approach

a Sliding diagram for a CCW pinched hysteresis loop with increasing excitation (green) and decreasing excitation (pink)

$\mathrm{b}$ Sliding diagram for $\mathrm{CW}$ pinched hysteresis loop under increasing excitation (green) and decreasing excitation (pink)

Initially, the value of both input $\alpha$ and output $\beta$ are assigned to be $0 . P$ and $Q$ are two points where line $A H$ and $B G$ intersect with the y axis shown in Fig.2a. The excitation function $\alpha(j)$ is assigned to move along the $\mathrm{x}$-axis of the sliding diagram, and such excitation sliding mechanism is defined for the situation 
This article has been accepted for publication in a future issue of this journal, but has not been fully edited.

Content may change prior to final publication in an issue of the journal. To cite the paper please use the doi provided on the Digital Library page. when the excitation is increasing and regardless of loop directions on the pinched hysteresis loop graph. The response value $\beta(j)$ is assigned to be the area value of rhombus $F_{j} E_{j} Q P$ at position $j$ after the excitation value is increasing from value $\alpha=0$ to $\alpha(j)$. The symbols in this case are highlighted with green colour in Fig.2a.

While at the point $j+1, \alpha(j+1)=M \cdot \sin [\omega \cdot \Delta T \cdot(j+1)]$. The incremental area $\Delta S_{j+1}$ between the position $j$ and $j+1$ is highlighted in green color. The response value (i.e. the area value) in position $j+1$ can be calculated by:

$$
\beta(j+1)=\beta(j)+\Delta S_{j+1}
$$

Equation (8) applies when an excitation is in a monotonic increasing condition.

When the periodic excitation is decreasing (i.e. $\left.\frac{d \alpha(j)}{d j}<0\right), \alpha(j)$ would move along the $y$ axis (instead of the x-axis). The response value (i.e. the area value) is highlighted in pink colour in Fig.2a. Since the magnitude of $\alpha(j)$ is decreasing, (8) is re-expressed as (9) when the excitation is in a monotonic decreasing condition.

$$
\beta(j+1)=\beta(j)-\Delta S_{j+1}
$$

\section{2) Response Generation of a CW Loop}

For a CW pinched hysteresis loop, (8) still applies when the excitation is increasing. But the incremental area $\Delta S_{j+1}$ should be obtained from rhombus $F_{j} E_{j} F_{j+l} E_{j+1}$ highlighted in green colour as shown in Fig.2b.

For decreasing excitation in a CW loop, (9) also applies. The incremental area can be determined from the rhombus highlighted in pink colour in the sliding diagram of Fig. $2 b$.

\subsection{Preliminary pinched hysteresis loop}

In this section, the graphical method is illustrated to explore the characteristic of the pinched hysteresis loop. The frequency-dependency of the pinched loop is explained.

1) Pinched at the origin

The following simulation is based on the parameters:

- $y_{G}$ and $y_{H}$ are assigned to be 0.4 and -0.4 for counter clockwise loop direction;

- $\quad x_{H}$ and $x_{G}$ are equal to -0.4 and 0.4 , respectively, for clockwise loop direction;

- the maximum value of excitation is 1 . 
This article has been accepted for publication in a future issue of this journal, but has not been fully edited.

Content may change prior to final publication in an issue of the journal. To cite the paper please use the doi provided on the Digital Library page.

The Lissajous figures of $\alpha$ and $\beta$ with different loop directions are shown in Fig.3a. It can be seen that the proposed method features the first fingerprint of hysteresis loops pinched at the origin. However, it can be noted that one side of the pinched hysteresis loop is a straight line which is not common to physical memristors. Such issue will be dealt with in Section 3.

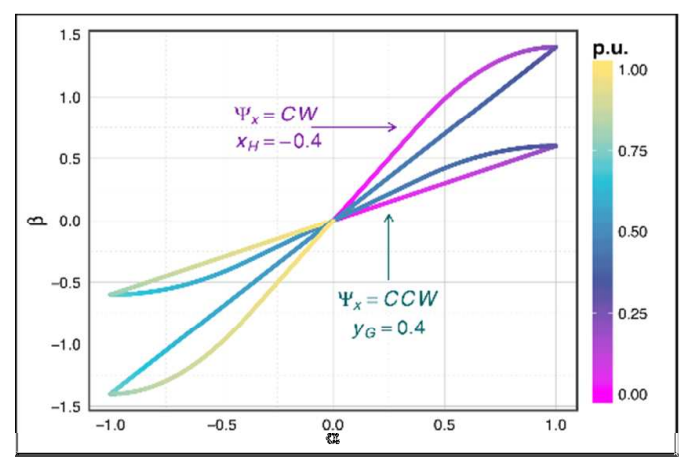

a

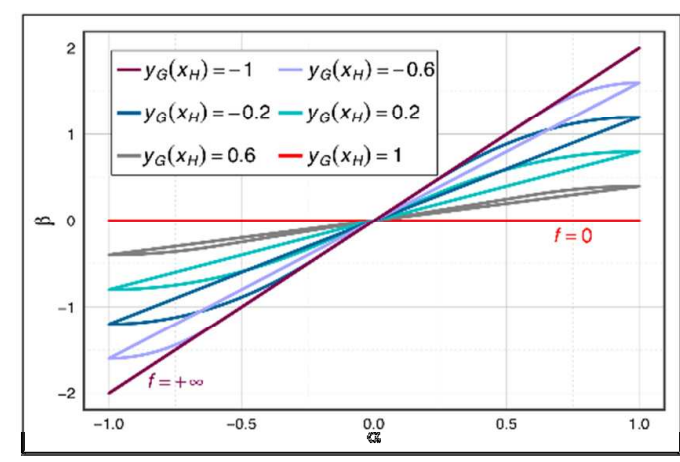

b

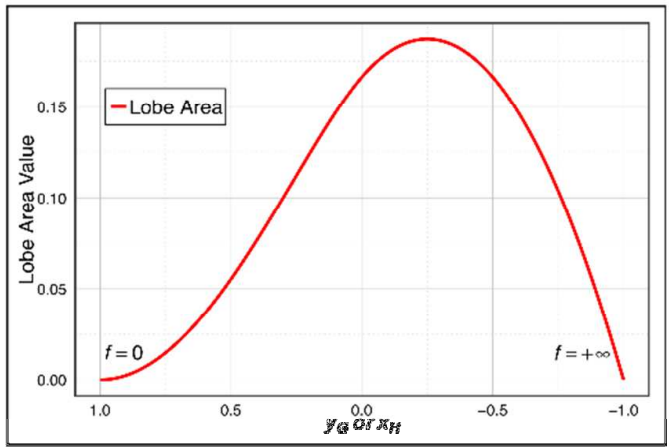

C

Fig. 3. Characteristics of the graphical approach generated pinched hysteresis loops a Plots of pinched hysteresis loops based on a range of $y_{G}$ and $x_{H}$ values $\mathrm{b}$ Plots of pinched hysteresis loops based on a range of $y_{G}$ and $x_{H}$ values c Typical variation of the lobe area based on the proposed method (with $y_{G}$ or $x_{H}=1$ representing dc operation and $y_{G}$ or $x_{H}=-1$ representing extreme high excitation frequency operation for a counter clockwise loop)

2) Frequency-dependent lobe areas

From Fig.3a, it can be seen that $y_{G}$ and $x_{H}$ affect the slopes of the pinched hysteresis loop. Therefore, $y_{G}$ or $x_{H}$ values can be used to represent different frequency operations of a memristor. Fig.3b shows a range of pinched hysteresis loop based on several sets of $y_{G}$ and $x_{H}$ values. In general, a large $y_{G}$ value represents low excitation frequency condition while a small $y_{G}$ high excitation frequency in $\mathrm{CCW}$ loop direction. Similarly, for the $\mathrm{CW}$ direction case, a large $x_{H}$ value represents low excitation frequency condition and a small $x_{H}$ means high excitation frequency. It should be noted in Fig. $3 \mathrm{~b}$ that the pinched hysteresis loop becomes a straight line at the extreme low and extreme high frequency. The loop area 
This article has been accepted for publication in a future issue of this journal, but has not been fully edited.

Content may change prior to final publication in an issue of the journal. To cite the paper please use the doi provided on the Digital Library page. depends on the operating frequency. Therefore, the values of $y_{G}$ and $x_{H}$ provide the means for accounting for the frequency dependency of the pinched hysteresis loops, which is the second fingerprint of a memristor.

\section{3) Lobe area characteristics}

In order to illustrate the characteristic that lobe area would decrease when excitation is beyond the threshold value, a simulation is conducted by varying $y_{G}$ or $x_{H}$. The variation of the lobe area in the first quadrant from $y_{G}=+1$ to -1 is shown in Fig.3c. It shows that the lobe area initially increases from zero at dc operation (with $y_{G}=+1$ ) with increasing operating frequency. After reaching a maximum value, it decreases with increasing frequency and becomes zero again at extreme high frequency (with $y_{G}=-1$ ). Therefore, this typical frequency-dependent lobe area is in agreement with the third fingerprint of a memristor.

\section{Graphical approach for modelling pinched hysteresis loops}

As pointed out previously, one side of the pinched hysteresis loop in Fig.3a is a straight line which is not a common feature of a memristor. In this section, a modulator $\sigma$ and a compensator $C$ are introduced to the basic model in order to enhance the features of the pinched hysteresis loop. The use of the modulator alone will modify the shape of the loop, but has the side effect of losing the pinched position. The compensator is therefore used to remove this side effect and ensures that the loop goes through the origin.

\subsection{Modulator $\sigma$ and Compensator $C$ for increasing and decreasing excitation function}

1) Modulator $\sigma_{p}$ for $\frac{d \alpha(j)}{d j} \geq \mathbf{0}$

A new parameter $\sigma_{p}$ is introduced to the original equation (8), where the subscript $p$ stands for positive excitation derivative. For the region where the slope of the excitation is positive (i.e. $\left.\frac{d \alpha(j)}{d j} \geq 0\right)$ so that:

$$
\beta(j+1)=\left[\beta(j)+\Delta S_{j+1}\right] \cdot \sigma_{p}(j+1)
$$


This article has been accepted for publication in a future issue of this journal, but has not been fully edited.

Content may change prior to final publication in an issue of the journal. To cite the paper please use the doi provided on the Digital Library page.

2) Modulator $\sigma_{n}$ and compensator $C$ for $\frac{d \alpha(j)}{d j}<\mathbf{0}$

For the region when the excitation function is decreasing (with the subscript $n$ representing a negative excitation derivative), the original equation (11) can be modified as (11).

$$
\beta(j+1)=\left[\beta(j)-C_{j+1} \cdot \Delta S_{j+1}\right] \cdot \sigma_{n}(j+1)
$$

Equations (10) and (11) are the core equations for the positive and negative slopes of the excitation function, respectively and they form the key relationships between the output and the input of the model. The derivation of compensators is presented in the appendix. The derivations of the modulators, $\sigma_{p}$ and $\sigma_{n}$ for physical memristors will be demonstrated in Section 5.

\subsection{Summary of the proposed graphical modelling approach memristor}

The procedure of the proposed graphical modelling method is summarized as follows: suppose $p_{0}$

is the point where $\frac{d \alpha(j)}{d j} \geq 0$ starts to be effective. In other words, $p_{0}$ is the lowest point of the periodic excitation $\alpha$ in Fig.1a the computational procedures are concluded in equations (12) and (13) comparing with two iterative equations (10) and (11).

From point $p_{0}$ to point $p_{0}+N / 2$ (where $p_{0}+N / 2=N_{l}$ ) according to Fig.1a, i.e. the half of the cycle within which the excitation function is increasing:

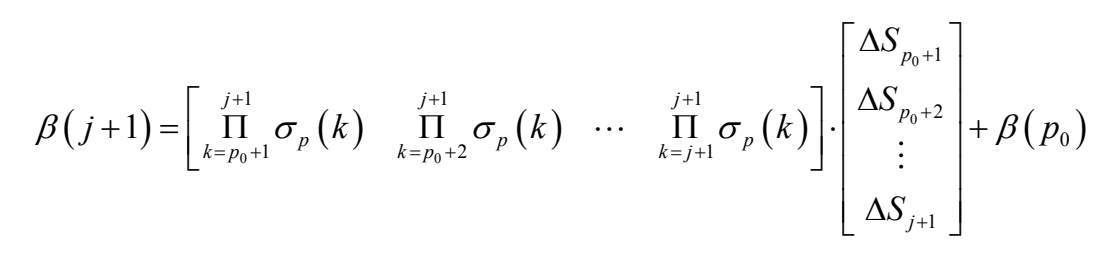

From point $p_{0}+N / 2+1$ (which can also be labelled as point $\left(N_{1}+1\right)$ ) to point $p_{0}+N$, i.e. the half of the cycle within which the excitation function is decreasing:

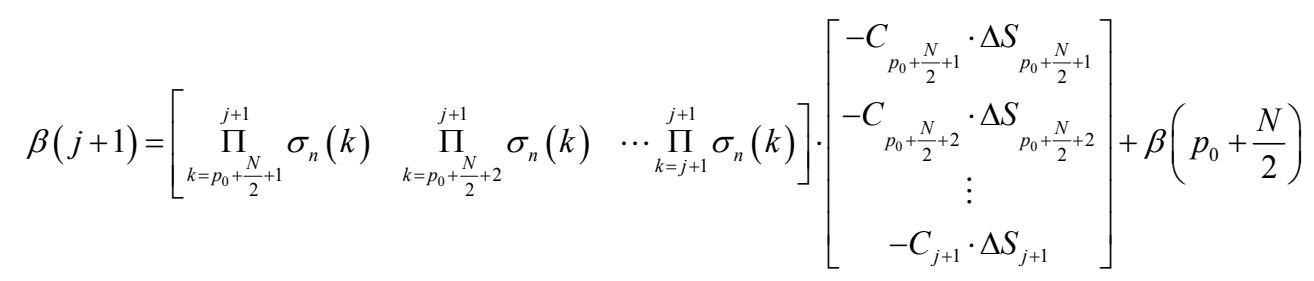

$\Pi$ is the factorial function in the equations, $k$ is function variable. Here are two examples of how factorial function operates:

$$
\prod_{k=p_{0}}^{j+1} \sigma_{p}(k)=\sigma_{p}\left(p_{0}\right) \cdot \sigma_{p}\left(p_{0}+1\right) \ldots \sigma_{p}(j) \cdot \sigma_{p}(j+1)
$$


This article has been accepted for publication in a future issue of this journal, but has not been fully edited. Content may change prior to final publication in an issue of the journal. To cite the paper please use the doi provided on the Digital Library page.

$$
\prod_{k=p_{0}+\frac{N}{2}+1}^{j+1} \sigma_{n}(k)=\sigma_{n}\left(p_{0}+\frac{N}{2}+1\right) \cdot \sigma_{n}\left(p_{0}+\frac{N}{2}+2\right) \ldots \cdot \sigma_{n}(j+1)
$$

In an actual memristor pinched hysteresis loop simulation process, excitation would typically starts from the origin at which the initial values are known. For the complementary part and considering the selfcrossing feature, the process would replicate what has been assigned for the first half of excitation.

Fig.4a and Fig.4b show how (12) and (13) are implemented over the regions of positive slope and negative slope of the excitation function, respectively. Equations (12) and (13) constitute the framework of the proposed graphical approach to the pinched hysteresis loop of memristor. A flow chart of the implementation of the implementation of this graphical method is illustrated in Fig.4c. It should be highlighted that a power amplification coefficient $\varepsilon$ is introduced. This coefficient is simply a constant for scaling the magnitude of the output so that the model can fit into a diversity of memristors.

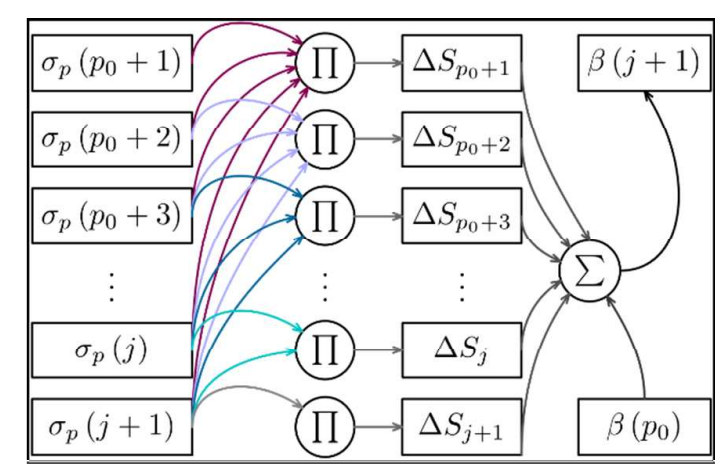

$a$

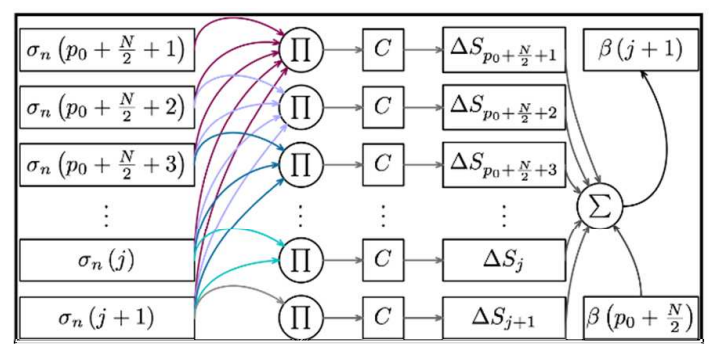

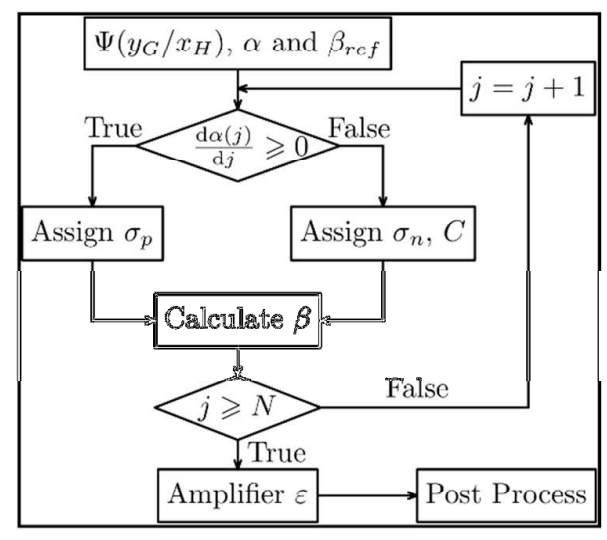

$c$

Fig. 4. Diagrams of the graphical approach

a Illustration of proposed graphical approach when the excitation function is increasing $\mathrm{b}$ Illustration of proposed graphical approach when the excitation function is decreasing $\mathrm{c}$ flowchart of the procedure of the proposed graphical modelling method

\section{Demonstration of the graphical methods for pinched hysteresis loops generation}

\subsection{Simulation of pinched hysteresis loop with different modulators value with $y_{G}=x_{H}=0$ for both CCW and CW directions}


This article has been accepted for publication in a future issue of this journal, but has not been fully edited.

Content may change prior to final publication in an issue of the journal. To cite the paper please use the doi provided on the Digital Library page.

New simulation plots with the parameter $y_{G}=0$ and for a range of modulator values (with $\sigma_{p}=\sigma_{n} \geq 1$ ) for CCW loops are displayed in Fig.5a. It can be seen that the modulator values can alter the shapes and hence the lobe areas of the pinched hysteresis loops. The larger the values of the modulators $(>1)$, the larger the loops become in this case.

For the CW loops and with $x_{H}=0$ and (with $\sigma_{p}=\sigma_{n} \leq 1$ ), a range of CW loops are plotted in Fig.5b. Unlike the CCW loops, the sizes of the $\mathrm{CW}$ loops increase with a decreasing value of the modulator $(<1)$ under such circumstances.

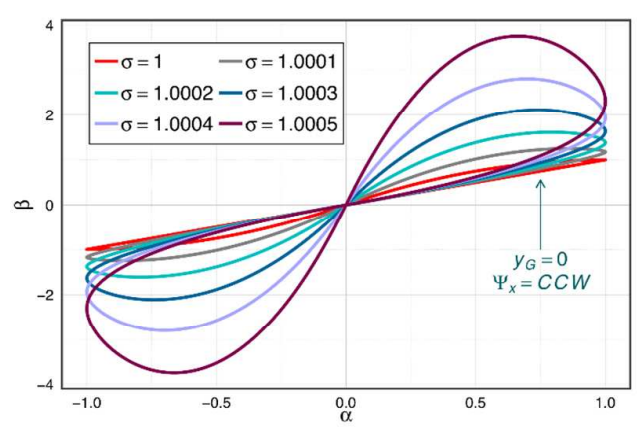

$a$

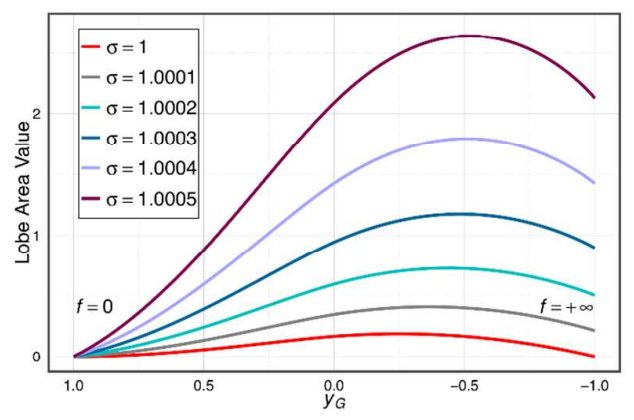

$c$
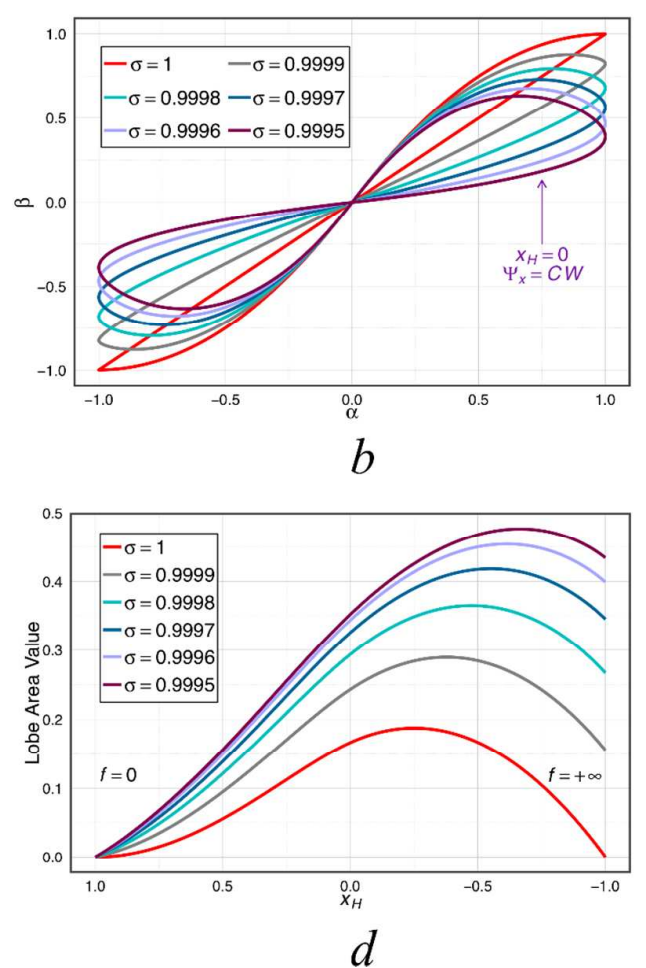

Fig. 5. Pinched hysteresis loop characteristics using the proposed graphical approach

a Plot of pinched hysteresis loops using proposed graphical approach for a range of modulator values while $y_{G}=0$ in $\mathrm{CCW}$ direction

$\mathrm{b}$ Plot of pinched hysteresis loops using proposed graphical approach with a variety of modulators while $x_{H}=0$ in $\mathrm{CW}$ direction c Plot of CCW pinched hysteresis loops lobe area curves in the first quadrant with a range of different modulator values $\mathrm{d}$ Plot of $\mathrm{CW}$ pinched hysteresis loops lobe area curves in the first quadrant with a range of different modulator values

\subsection{Lobe area analysis in the first quadrant with various $y_{G}$ and $x_{H}$ modulator and coefficient values assigned}

In Section II, it has been pointed out that the values of $y_{G}$ and $x_{H}$ can be used to alter the pinched hysteresis loops at different frequencies. These features have been evaluated by plotting the lobe areas in the first quadrant with their respective values for the $\mathrm{CCW}$ loops and the $\mathrm{CW}$ loops. Fig.5c shows the variations of the lobe areas for the $\mathrm{CCW}$ loops for a range of modulator values from 1 to 1.0005 . These 
This article has been accepted for publication in a future issue of this journal, but has not been fully edited.

Content may change prior to final publication in an issue of the journal. To cite the paper please use the doi provided on the Digital Library page.

curves are consistent in a sense that they approach zero at dc (represented by $y_{G}=1$ ), increase as $y_{G}$ decreases (i.e. increasing frequency). For the CW loops, the corresponding results are plotted in Fig.5d.

\section{HP memristor model simulation}

In this part, a series of simulations on HP memristor model using the introduced graphical model is presented. The HP memristor model [2] was developed in HP Lab in 2008.

Two platinum parts are attached on both sides of the semiconductor materials. The HP model has both highly doped and undoped region with a length of $w$ and $D-w$ respectively. Ammeter and voltmeter are attached to measure the current and the voltage for analysis. To assure that such device is working at a normal condition, the length of highly doped region should be restricted to $0<w<D$.

According to the explanation of such classical memristor model [2], the relationship between instantaneous voltage and current on memristor, the width of doped area and the charge are presented in the following equations:

$$
\left\{\begin{array}{c}
v(t)=\left[R_{O F F}\left(1-\frac{w(t)}{D}\right)+R_{O N} \frac{w(t)}{D}\right] \cdot i(t) \\
w(t)=\mu_{V} \cdot \frac{R_{O N}}{D} \cdot q(t)
\end{array}\right.
$$

where $i(t)$ and $v(t)$ are the current and voltage respectively; $q(t)$ is the electric charge at the current situation. $R_{O N}$ and $R_{O F F}$ are the resistance values when $w=D, w=0$ respectively. Furthermore, an average ion mobility parameter $\mu_{V}$ in the HP memristor model is assigned for simplicity.

\subsection{Modulator data generation}

In this paper, modulator data for generic memristor pinched hysteresis loops simulation purpose is generated in the following equations based on the following excitation scenarios:

1) $\quad \alpha\left(t_{j+1}\right) \neq 0$

$$
\sigma_{\mathrm{X}}\left(t_{j+1}\right)=1+\frac{\alpha\left(t_{j+1}\right)}{\left|\alpha\left(t_{j+1}\right)\right|} \cdot\left[(\lambda-1) \cdot \Delta_{\mathrm{X}}+\mu_{\mathrm{X}} \cdot\left(\frac{d \beta_{r e f}\left(t_{j+1}\right)}{d t}\right)^{\lambda}\right]
$$

2) $\quad \alpha\left(t_{j+1}\right)=0$

$$
\sigma_{\mathrm{x}}\left(t_{j+1}\right)=1+\frac{\alpha\left(t_{j}\right)}{\left|\alpha\left(t_{j}\right)\right|} \cdot\left[(\lambda-1) \cdot \Delta_{\mathrm{X}}+\mu_{\mathrm{X}} \cdot\left(\frac{d \beta_{r e f}\left(t_{j+1}\right)}{d t}\right)^{\lambda}\right]
$$

In the above two equations: $\sigma$ and $\mu$ represent the modulators and parameter controlling the proportion of corresponding reference output derivative values $\beta_{\text {ref }}$ for both excitation increasing and decreasing 
This article has been accepted for publication in a future issue of this journal, but has not been fully edited.

Content may change prior to final publication in an issue of the journal. To cite the paper please use the doi provided on the Digital Library page.

situations; $\Delta$ is parameter added to leverage the modulators horizontally; $\lambda$ is an integer to control exponential of the referenced derivative values and the existence of $\Delta$; the subscript $X$ depicts the condition of excitation derivative, subscripts would be $p$ or $n$ if $X \geq 0$ or $X<0$.

\subsection{Comparisons between theoretical HP model and graphical model}

The Matlab based source code of the mathematic model of HP memristor is given in [21] and the generated data serve as the reference. The generated pinched hysteresis loop is considered as the theoretical HP memristor pinched hysteresis loop. The results of the HP model can be used for determining the essential parameters of the proposed graphical approach. A table containing the parameter settings is presented in Table 1 .

Table 1 Parameter settings for HP theoretical memristor model

\begin{tabular}{cccc}
\hline \multicolumn{2}{c}{ HP Theoretical Model } & \multicolumn{2}{c}{ Graphical Model } \\
\hline item & value & item & value \\
\hline$\alpha(t)$ & $v_{0} \cdot \sin \left(\omega_{0} t\right)$ & $y_{G}$ & -0.387 \\
$R_{O N}$ & $100 \Omega$ & $\varepsilon$ & $6 \times 10^{-5}$ \\
$R_{O F F}$ & $1.6 \times 10^{4} \Omega$ & $\mu_{p}$ & 0.139 \\
$R_{\text {init }}$ & $1.6 \times 10^{4} \Omega$ & $\Delta_{p}$ & $1.92 \times 10^{-4}$ \\
$\alpha$ & $1 \times \sin (2 \pi \cdot \mathrm{t})$ & $\mu_{n}$ & 0.1 \\
$D$ & $10 \mathrm{~nm}$ & $\Delta_{n}$ & $9.28 \times 10^{-5}$ \\
$\mu_{V}$ & $1 \times 10^{-14}$ & $\lambda$ & 2 \\
\hline
\end{tabular}

In Table I, for the HP memristor model, $R_{O N}$ and $R_{O F F}$ are the largest and smallest resistance according to boundary condition; $R_{\text {init }}$ is the initial memristance value. There are 7 items in the graphical modelling schema settings: $y_{G}$ indicates the loop direction yet defines frequency related parameter; amplification $\varepsilon$ is assigned to adapt the scale while different memristor models; $\mu_{p}, \Delta_{p}, \mu_{n}, \Delta_{n}$ as well as $\lambda$ are the parameters in equations (15) and (16) used to calculate the modulator values.

Pinched hysteresis loops based on the memristor theoretical model and the proposed graphical approach of memristor are plotted in Fig.6a for comparison for an excitation of $1 \mathrm{~Hz}$ pure sinusoidal voltage waveform. It can be seen that the pinched hysteresis loops essentially overlap. Fig.6b shows the response current waveforms of the two methods. Again, they have very good agreement because both waveforms essentially overlap. Although the HP model does not need a modulator, such parameter can be reconstructed from the simulation results and compared with the modulator of the graphical method as shown in Fig.6c. The blue line is generated by the equations (15) and (16) while the brown line is reconstructed with the data method using HP theoretical model. 
This article has been accepted for publication in a future issue of this journal, but has not been fully edited. Content may change prior to final publication in an issue of the journal. To cite the paper please use the doi provided on the Digital Library page.

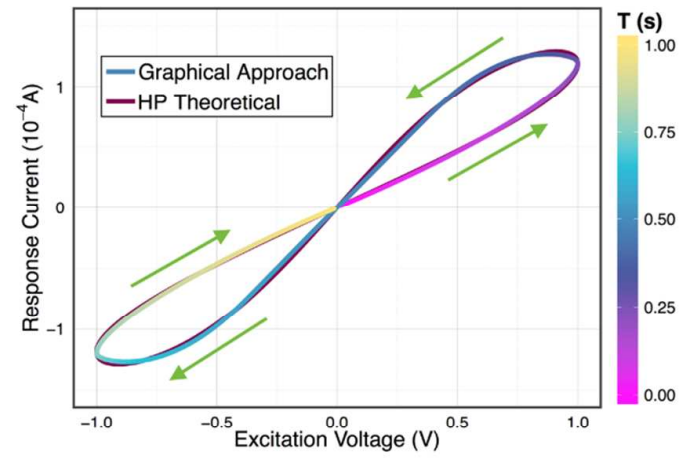

$a$

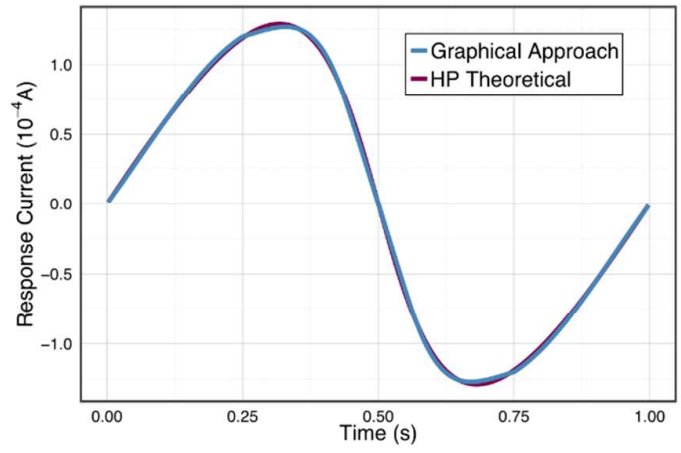

$b$

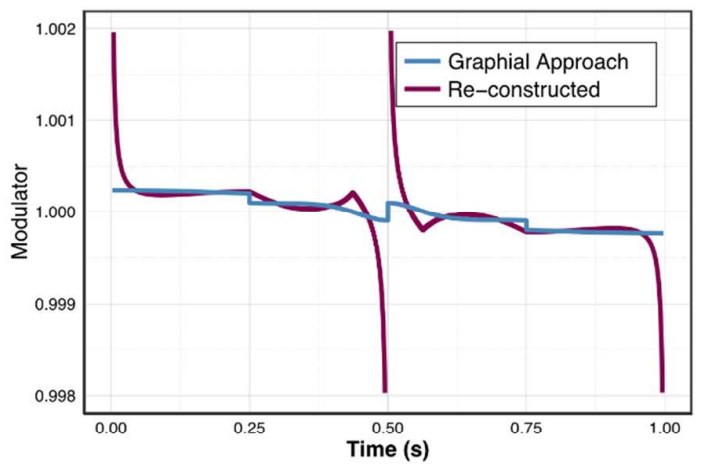

C

Fig. 6. HP theoretical model related demonstration figures

a The comparison between theoretical HP memristor model and graphical approach

b Response current waveform comparison between HP theoretical model and graphical model

c Modulators comparison of HP theoretical memristor

\subsection{Comparisons of experimental data and the graphical approach of HP experimental memristor characteristics}

In this set of simulations, a sinusoidal voltage is applied to the HP memristor, the frequency of the excitation voltage waveform is $1 / 200 \mathrm{~Hz}$. Sampling frequency for HP experimental simulations is set to 10 $\mathrm{kHz}$ which is the same with the previous HP theoretical model.

1) HP experimental model with single pinched hysteresis loop

Considering the actual HP experimental memristor model, the specific parameters of such nanoscale device and graphical model optimized parameters are all presented completely in the following Table 2 according to [2]. Both the setup of the HP memristor and the optimized parameters of graphical model generated by the genetic algorithm are listed on the left and right side of the table.

Table 2 Specifications of graphical model for triple pinched hysteresis loops HP memristor 
This article has been accepted for publication in a future issue of this journal, but has not been fully edited.

Content may change prior to final publication in an issue of the journal. To cite the paper please use the doi provided on the Digital Library page.

\begin{tabular}{cccc}
\hline \multicolumn{2}{c}{ HP Experimental Setup } & \multicolumn{2}{c}{ Graphical Model } \\
\hline item & value & item & value \\
\hline$\alpha(t)$ & $\mathrm{v} 0 \cdot \sin (\omega 0 \mathrm{t})$ & $y_{G}$ & $8.5 \times 10-3$ \\
$v 0$ & $1 \mathrm{~V}$ & $\varepsilon$ & $6.8 \times 10-3$ \\
$i 0$ & $10 \mathrm{~mA}$ & $\mu p$ & $6.9 \times 10-5$ \\
$t 0$ & $10 \mathrm{~ms}$ & $\Delta p$ & $2.0 \times 10-4$ \\
$D$ & $10 \mathrm{~nm}$ & $\mu n$ & $7.0 \times 10-1$ \\
$\mu V$ & $10-10$ & $\Delta n$ & $3.3 \times 10-4$ \\
$R O F F / R O N$ & 160 & $\lambda$ & 2 \\
\hline
\end{tabular}

According to Table 2, $\alpha$ is the excitation equation; $\omega_{0}$ is angular speed equals to $\pi / 100 \mathrm{rad} / \mathrm{s} ; v_{0}$ is the max value of excitation voltage; $i_{0}$ is calculated by $v_{0} / R_{O N}$ which is the max current through the device; $t_{0}$ is shortest time for dopants to drift linearly through the total length of device; $D$ is the total length of the total memristive device; $\mu_{V}$ is the average ion mobility in a uniform electric field.

On the right side of the table, seven parameters optimized by the genetic algorithm are listed which are used in graphical modelling procedure: $y_{G}$ determines the loop direction and relates to the excitation frequency of the simulation; $\varepsilon$ is a unified magnification value of graphical model; $\mu_{p}, \Delta_{p}, \mu_{n}, \Delta_{n}$ and $\lambda$ are the parameters for generating modulators in equations (15) and (16).

By optimizing the parameters of the introduced graphical approach with genetic algorithm, the results for both experimental data and the graphical model are shown as follows including pinched hysteresis loop, current waveforms as well as modulator comparisons.

Fig.7a displays the measured pinched loop of the HP memristor and the result obtained by the graphical method. The corresponding response currents are plotted in Fig.7b In both cases, the measurements and the graphical results have very good agreements. The modulator variation reconstructed from the practical measurements can be compared with that of the graphical method as shown in Fig.7c. These results confirm that the graphical method provides the appropriate framework to model the pinched hysteresis loop. 
This article has been accepted for publication in a future issue of this journal, but has not been fully edited. Content may change prior to final publication in an issue of the journal. To cite the paper please use the doi provided on the Digital Library page.
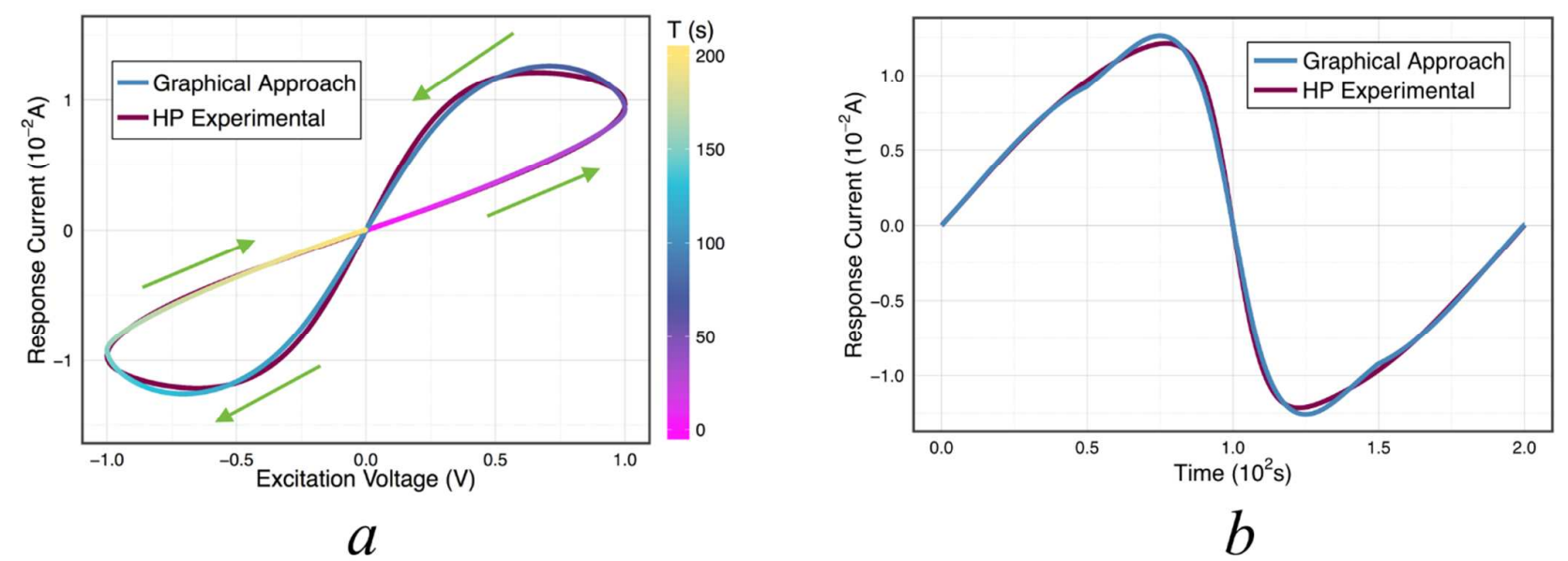

$a$

$b$

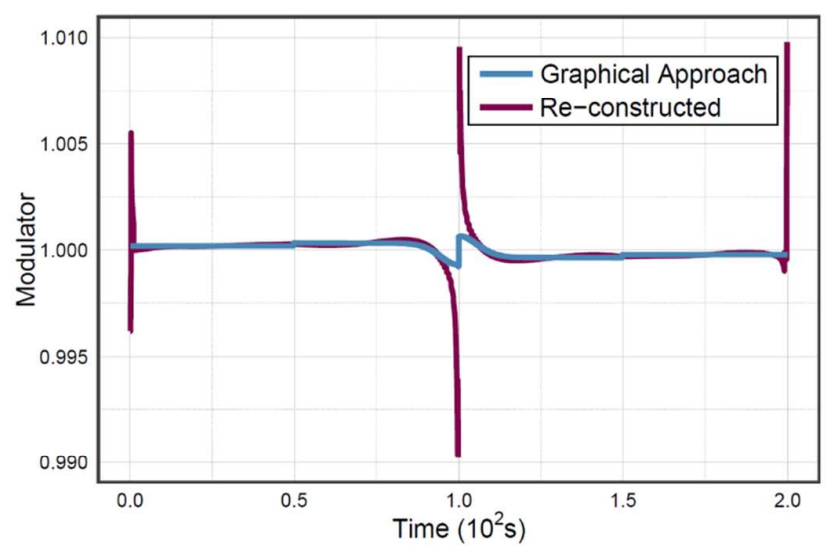

C

Fig. 7. HP experimental single loop based figures

a Comparison between the measured pinched loop of the HP memristor and the result of the graphical method

$\mathrm{b}$ Comparison of the response current between HP memristor and the graphical model

c Modulator function of the graphical approach and the corresponding function reconstructed from measurements

2) HP experimental model tested with triple pinched hysteresis loops

For the tests of the HP experimental model with triple pinched hysteresis loops, the parameters are the same as shown in Table 2 except that: $\alpha(t)= \pm v_{0} \cdot \sin \left(\omega_{0} t\right)$, total simulation time is $600 \mathrm{~s}$ and $R_{O F F} / R_{O N}=380$.

Parameter settings of graphical model for triple loops HP experimental memristor are presented in Table 3. Note that there are six half cycles of the excitation voltage waveforms enumerated from 1 to 6 for reference [2].

Table 3 Specifications of Graphical Model for Triple Pinched Hysteresis Loops HP Memristor

\begin{tabular}{cccc}
\hline Half-cycle & $1 \& 6$ & $2 \& 5$ & $3 \& 4$ \\
\hline$y_{G}$ & $8.5 \times 10-3$ & $8.5 \times 10-3$ & $8.5 \times 10-3$ \\
$\varepsilon$ & $2.72 \times 10-3$ & $3 \times 10-3$ & $4.3 \times 10-3$ \\
\hline
\end{tabular}


This article has been accepted for publication in a future issue of this journal, but has not been fully edited.

Content may change prior to final publication in an issue of the journal. To cite the paper please use the doi provided on the Digital Library page.

\begin{tabular}{cccc}
$\mu_{p}$ & $1 \times 10-6$ & $8 \times 10-5$ & $1 \times 10-6$ \\
$\Delta p$ & $1.7 \times 10-4$ & $1.4 \times 10-4$ & $3.5 \times 10-4$ \\
$\mu_{n}$ & $5.5 \times 10-2$ & $3 \times 10-5$ & $1 \times 10-6$ \\
$\Delta n$ & $-3.3 \times 10-4$ & $-4 \times 10-5$ & $1.6 \times 10-4$ \\
$\lambda$ & 2 & 2 & 2 \\
\hline
\end{tabular}

The excitation frequency value throughout the HP experimental memristor simulation study is unchanged. $y_{G}$ is selected to be $8.5 \times 10^{-3}$ which is the same as the single pinched hysteresis loops case. The amplification parameters $\varepsilon$ are different according to the accumulation of charge in the device. While the next 4 parameters are used to calculate the graphical model modulator values, it should be noted that the parameter $\lambda$ is 2 .

The practical measurements of the HP device (captured from [2]) and the simulation results are plotted in Fig.8a. The corresponding response current waveforms are shown in Fig.8b. These results confirm that the graphical approach can be used to model the self-crossing pinched hysteresis loops of a memristor. The modulator function of the graphical method and the reconstructed one from the measurements are displayed in Fig.8c. The modulator function of the graphical approach is a theoretical concept with the discontinuity issue.

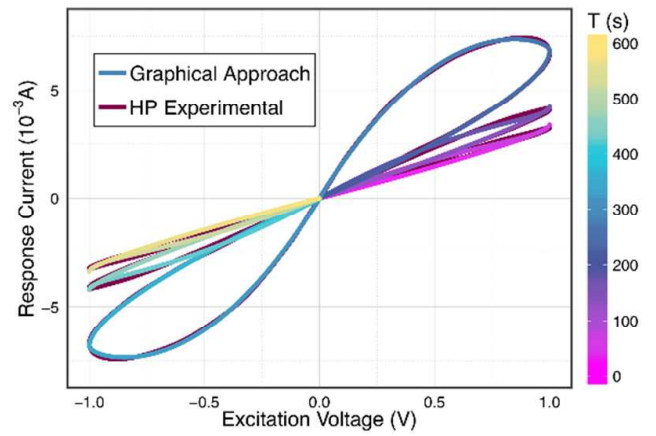

$a$

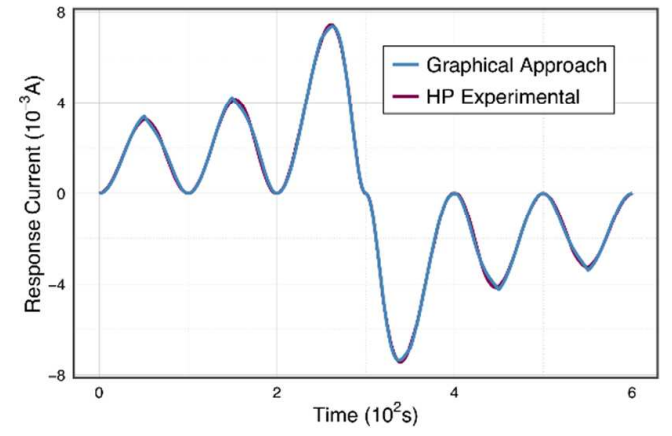

$b$

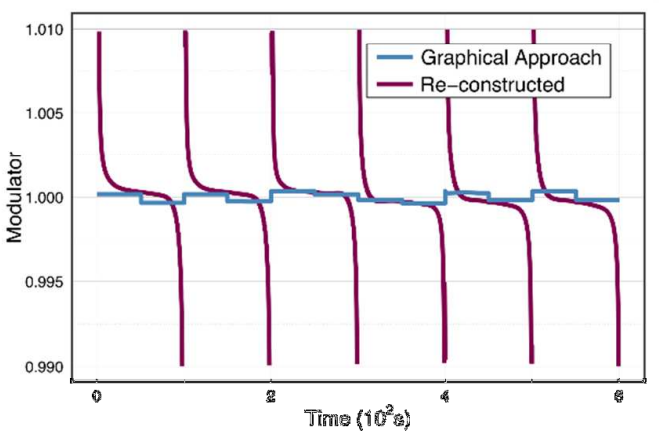

ce

Fig. 8. HP triple loops experimental case based figures 
This article has been accepted for publication in a future issue of this journal, but has not been fully edited.

Content may change prior to final publication in an issue of the journal. To cite the paper please use the doi provided on the Digital Library page.

a Comparison between the measured pinched loop of the HP memristor and the result of the graphical method (triple pinched hysteresis loops)

b Response current comparison between graphical approach and HP experimental memristor with triple pinched hysteresis loops.

c Modulator comparison graph of HP experimental memristor triple pinched hysteresis loops

\section{Conclusion}

This paper presents a new graphical method for modelling pinched hysteresis loops exhibited by memristors. The shape of the loops can be modified with the aids of a modulator and a compensator. The frequency-dependent aspects of the loops can be modified with the values of a parameter $y_{G}$ (for CCW loops) and $x_{H}$ (for CW loops). The algorithms and procedures of the proposed method have been illustrated. The results show that the proposed graphical method can be used to generate pinched hysteresis loops that exhibit the three fingerprints of a memristor. The HP memristor model is used as reference to evaluate the graphical model performance.

\section{Appendices}

Compensator is used to keep the hysteresis loop pinched at the origin during the decreasing excitation conditions. Let the nadir point be $p_{0}$. The final response value at excitation increasing situation can be represented with $\beta\left(p_{0}+\frac{N}{2}\right)$, where $N$ is the total sampling number in one excitation cycle period. The next response value should be $\beta\left(p_{0}+\frac{N}{2}+1\right)$ based on decreasing excitation condition.

In order to assure that the hysteresis loop should be pinched at the origin, the response value $\beta\left(p_{0}+\frac{3 N}{4}\right)$ needs to be 0 at the excitation value: $\alpha\left(p_{0}+\frac{3 N}{4}\right)$.

According to (13) and (15) for decreasing excitation conditions, the following iterative equations related to $\beta\left(p_{0}+\frac{3 N}{4}\right)$ can be developed:

$$
\begin{aligned}
& \beta\left(p_{0}+\frac{3 N}{4}\right)=\left[\beta\left(p_{0}+\frac{3 N}{4}-1\right)-C \cdot \Delta S_{p_{0}+\frac{3 N}{4}}\right] \cdot \sigma_{n}\left(p_{0}+\frac{3 N}{4}\right) \\
& \beta\left(p_{0}+\frac{3 N}{4}\right)=\left[\beta\left(p_{0}+\frac{3 N}{4}-2\right)-C \cdot \Delta S_{p_{0}+\frac{3 N}{4}-1}\right] \cdot \sigma_{n}\left(p_{0}+\frac{3 N}{4}-1\right) \cdot \sigma_{n}\left(p_{0}+\frac{3 N}{4}\right)-C \cdot \Delta S_{p_{0}+\frac{3 N}{4}-1} \cdot \sigma_{n}\left(p_{0}+\frac{3 N}{4}\right)
\end{aligned}
$$

Based on the iterative equations stated above, the relationship between $\beta\left(p_{0}+\frac{3 N}{4}\right)$ and $\beta\left(p_{0}+\right.$ $\left.\frac{N}{2}\right)$ is presented as follows: 
This article has been accepted for publication in a future issue of this journal, but has not been fully edited.

Content may change prior to final publication in an issue of the journal. To cite the paper please use the doi provided on the Digital Library page.

$$
\beta\left(p_{0}+\frac{3 N}{4}\right)=\beta\left(p_{0}+\frac{N}{2}\right)-C \cdot \Delta S_{p_{0}+\frac{3 N}{4}} \cdot \sigma_{n}\left(p_{0}+\frac{3 N}{4}\right) \cdots-C \cdot \Delta S_{p_{0}+\frac{N}{2}+1} \cdot \underbrace{p_{0}+\frac{3 N}{4}}_{k=p_{0}+\frac{N}{2}+1} \sigma_{n}(k)-C \cdot \Delta S S_{p_{0}+\frac{N}{2}} \cdot \prod_{k=p_{0}+\frac{N}{2}}^{p_{0}+\frac{3 N}{4}} \sigma_{n}(k)
$$

Based on the definition of pinched hysteresis loop of memristor, the response value $\beta\left(p_{0}+\frac{3 N}{4}\right)=$ 0 is required for pinching at the origin, that's the reason why the compensation parameter $C$ is introduced in the graphical approach. Equation (19) could be derived as:

$$
\beta\left(p_{0}+\frac{N}{2}\right)=C \cdot \Delta S_{p_{0}+\frac{3 N}{4}} \cdot \sigma_{n}+C \cdot \Delta S S_{p_{0}+\frac{3 N}{4}-1} \cdot \prod_{k=p_{0}+\frac{3 N}{4}-1}^{p_{0}+\frac{3 N}{4}} \sigma_{n}(k)+\cdots \cdots+C \cdot \Delta S \sum_{p_{0}+\frac{N}{2}+1} \cdot \underset{k=p_{0}+\frac{N}{2}+1}{\prod_{0}+\frac{3 N}{4}} \sigma_{n}(k)+C \cdot \Delta S \underset{p_{0}+\frac{N}{2}}{\prod_{k=p_{0}+\frac{N}{2}}^{p_{0}+\frac{3 N}{4}}} \sigma_{n}(k)
$$

Now, a symbol $\Sigma_{C}$ is introduced in order to calculate the compensator $C$ value. $\Sigma_{C}$ is presented in equation (21).

$$
\Sigma_{C}=\Delta S_{p_{0}+\frac{3 N}{4}} \cdot \sigma_{n}\left(p_{0}+\frac{3 N}{4}\right)+\Delta S_{p_{0}+\frac{3 N}{4}-1} \cdot \underbrace{p_{0}+\frac{3 N}{4}}_{k=p_{0}+\frac{3 N}{4}-1} \sigma_{n}(k)+\cdots+\Delta S_{p_{0}+\frac{N}{2}+1} \cdot \prod_{k=p_{0}+\frac{N}{2}+1}^{\prod_{0}+\frac{3 N}{4}} \sigma_{n}(k)+\Delta S_{p_{0}+\frac{N}{2}} \cdot \prod_{k=p_{0}+\frac{N}{2}}^{\prod_{0}+\frac{3 N}{4}} \sigma_{n}(k)
$$

From (20) and (21)

$$
C=\frac{\beta\left(p_{0}+\frac{N}{2}\right)}{\Sigma_{C}}
$$

where $\beta\left(p_{0}+\frac{N}{2}\right)$ is the final value generated in the increasing excitation scenarios.

\section{References}

[1] L. Chua, "Memristor- The missing circuit element," IEEE Transactions on Circuit Theory, vol. 18, pp. 507-519, 1971.

[2] D. B. Strukov, G. S. Snider, D. R. Stewart, and R. S. Williams, "The missing memristor found," Nature, vol. 453, pp. 80-83, May 12008.

[3] L. O. Chua, "The Fourth Element," Proceedings of the IEEE, vol. 100, pp. 1920-1927, 2012.

[4] S. P. Adhikari, M. P. Sah, H. Kim, and L. O. Chua, "Three Fingerprints of Memristor," IEEE Transactions Circuits and Systems I: Regular Papers, on, vol. 60, 2013, pp. 3008-3012.

[5] M.P. Sah, H. Kim and L.O. Chua, "Brains are made of memristors", IEEE Circuits \& Systems Magazine, vo.14, pp: 12-36, 2014

[6] L.O. Chua, "Memristor, Hodgkin-Hurley and edge of chaos", Nanotechnology, vol.2, 383001, pp:1-14, 2013

[7] A. Volkov, C. Tucket, J. Reedus, M. Volkova, V. Markin andL. Chua, "Memristors in plants", Plant Signal Behavior, vol 9,. Feb 2014, pp: 28152-1 to 25182-8

[8] T. Prodromakis, C. Toumazou, and L. Chua, "Two centuries of memristors," Nature Materials, vol. 11, pp. 478481, 06//print 2012

[9] F. Corinto and A. Ascoli, "Memristive diode bridge with LCR filter," Electronics Letters, vol. 48, pp. 824-825, 2012.

[10] D. Lin, L.O. Chua and S.Y.R. Hui, "The first man-made memristor: circa 1801", Proceedings of the IEEE, (to appear) 
This article has been accepted for publication in a future issue of this journal, but has not been fully edited.

Content may change prior to final publication in an issue of the journal. To cite the paper please use the doi provided on the Digital Library page.

[11] H. Davy, "Additional experiments on Galvanic electricity, in a letter to Mr. Nicholson, dated September 22, 1800," (Nicholson's) Journal of Natural Philosopy, Chemistry, and the Arts, vol. IV, pp. 326-328, 1801.

[12] D.C. Jiles and D.L. Atherton, "Ferromagnetic hysteresis", IEEE Transactions on Magnetics, Volume: 19, Issue: 5, 1983 , Page(s): $2183 \quad-2185$

[13] F. Preisach, "Uber die magnetische nachwirkung", Zeitschrift fur Physik, 94, 1935, pp: 277-303

[14] R.M. Del Vecchio, "An efficient procedure for modeling complex hysteresis process in ferromagnetic materials", IEEE Transactions on Magnetics, Vol. MAG-16, No. 5, Sept, 1980, pp: 809-811

[15] P. Ivanyi, "Computer realization of the continuous Preisach hysteresis model", Technical Gazette 20, 2013, pp:329-334

[16] J.G. Zhu, S.Y.R. Hui, and V.S. Ramsden, 'A generalized dynamic circuit model of magnetic cores for low- and high-frequency applications. I. Theoretical calculation of the equivalent core loss resistance', IEEE Transactions on Power Electronics, Volume: 11 Issue: 2, March 1996, Page(s): $246-250$

[17] S.Y.R. Hui, J.G. Zhu, and V.S. Ramsden, 'A generalized dynamic circuit model of magnetic cores for low- and high-frequency applications. II. Circuit model formulation and implementation', IEEE Transactions on Power Electronics, Volume: 11 Issue: 2, March 1996, Page(s): 251 -259

[18] D. Lin, S.Y.R. Hui and L.O. Chua, "Gas discharge lamps are volatile memristors", IEEE Transactions on Circuits \& Systems I, Vol.61, No.7, 2014, pp: 2066-2073

[19] W. Dghais, L. Alves, J. Mendes and J. Rodriguez, "Memristor state-space embedding", European Conference on CIrcuit Theory and Design, 2015, 24-26 Aug. 2015, Trondheim, pp:1-4.

[20] S. P. Adhikari, M. P. Sah, H. Kim, and L. O. Chua, "Three Fingerprints of Memristor," IEEE Transactions on Circuits and Systems I: Regular Papers, vol. PP, pp. 1-14, 2013.

[21] K. Zaplatilek, "Memristor modeling in MATLAB and Simulink" in Proceedings of the European computing conference, 2011, pp. 62-67. 